IJMMS 30:10 (2002) 593-604

PII. S0161171202108283

http://ijmms.hindawi.com

(c) Hindawi Publishing Corp.

\title{
COMPLETELY GENERALIZED MULTIVALUED NONLINEAR QUASI-VARIATIONAL INCLUSIONS
}

\author{
ZEQING LIU, LOKENATH DEBNATH, SHIN MIN KANG, \\ and JEONG SHEOK UME
}

Received 10 August 2001

\begin{abstract}
We introduce and study a new class of completely generalized multivalued nonlinear quasivariational inclusions. Using the resolvent operator technique for maximal monotone mappings, we suggest two kinds of iterative algorithms for solving the completely generalized multivalued nonlinear quasi-variational inclusions. We establish both four existence theorems of solutions for the class of completely generalized multivalued nonlinear quasivariational inclusions involving strongly monotone, relaxed Lipschitz, and generalized pseudocontractive mappings, and obtain a few convergence results of iterative sequences generated by the algorithms. The results presented in this paper extend, improve, and unify a lot of results due to Adly, Huang, Jou-Yao, Kazmi, Noor, Noor-Al-Said, Noor-Noor, Noor-Noor-Rassias, Shim-Kang-Huang-Cho, Siddiqi-Ansari, Verma, Yao, and Zhang.
\end{abstract}

2000 Mathematics Subject Classification: 47J20, 49J40.

1. Introduction. In 1996, Adly [1] used the resolvent operator technique for maximal monotone mapping to study a general class of variational inclusions with singlevalued mappings. Afterwards, Huang [4] and M. A. Noor [10] extended this technique for a completely general class of variational inclusions with set-valued mappings and a class of general set-valued variational inclusions with compact-valued mappings, respectively. Recently, Shim et al. [14] extended the results in [1, 4, 10] to the generalized set-valued strongly nonlinear quasi-variational inclusions without compactness.

In this paper, we first introduce a new class of completely generalized multivalued nonlinear quasi-variational inclusions for multivalued mappings. Motivated and inspired by the methods of Aldy [1], Huang [4], M. A. Noor [10], and Shim et al. [14], we construct two new iterative algorithms for solving the completely generalized multivalued nonlinear quasi-variational inclusions with bounded closed valued mappings. We also establish four existence theorems of solutions for the class of completely generalized multivalued nonlinear quasi-variational inclusions involving strongly monotone, relaxed Lipschitz and generalized pseudocontractive multivalued mappings, and give some convergence results of iterative sequences generated by the algorithms. Our results extend, improve and unify a lot of results due to Adly [1], Huang [2, 3, 4], Jou and Yao [5], Kazmi [6], M. A. Noor [8, 9, 10], M. A. Noor and Al-Said [11], M. A. Noor and K. I. Noor [12], M. A. Noor et al. [13], Shim et al. [14], Siddiqi and Ansari [15, 16], Verma [18, 19], Yao [20], and Zhang [21].

2. Preliminaries. Let $H$ be a real Hilbert space endowed with a norm $\|\cdot\|$ and an inner product $\langle\cdot, \cdot\rangle, 2^{H}$, and $C B(H)$ denote the families of all nonempty subsets and 
all nonempty bounded closed subsets of $H$, respectively. Let $I$ stand for the identity mapping on $H$, and $H(\cdot, \cdot)$ be the Hausdorff metric on $C B(H)$.

Given single-valued mappings $g, h: H \rightarrow H$, multivalued mappings $A, B, C, D, E$ : $H \rightarrow 2^{H}$ and nonlinear mappings $N, M: H \times H \rightarrow H$. Suppose that $W: H \rightarrow 2^{H}$ is a maximal monotone mapping and $f \in H$. We consider the following problem.

Find $u \in H, x \in A u, y \in B u, z \in C u, v \in D u, w \in E u$ such that $g u-h w \in$ $\operatorname{dom}(W)$ and

$$
f \in N(x, y)-M(z, v)+W(g u-h w),
$$

which is called completely generalized multivalued nonlinear quasi-variational inclusion.

It is known that the subdifferential of a proper convex lower semicontinuous function is a maximal monotone mapping. But the converse is not true.

SPECIAL CASES. (i) If $f=0, C=D=I$, and $M(x, x)=0$ for all $x \in H$, then problem (2.1) is equivalent to finding $u \in H, x \in A u, y \in B u, w \in E u$ such that $g u-h w \in$ $\operatorname{dom}(W)$ and

$$
0 \in N(x, y)+W(g u-h w)
$$

which is called the generalized set-valued strongly nonlinear quasi-variational inclusion, studied by Shim et al. [14].

(ii) If $f=h=0, C=D=E=I, M(x, x)=0$ for all $x \in H$, then problem (2.1) collapses to finding $u \in H, x \in A u, y \in B u$ such that $g u \in \operatorname{dom}(W)$ and

$$
0 \in N(x, y)+W(g u)
$$

which is known as the general set-valued variational inclusion, introduced and studied by Noor [10].

(iii) If $f=g=0, C=D=I, M(x, x)=0, N(x, y)=a x-b y, c x=-h x$ for all $x, y \in H$, where $a, b: H \rightarrow H$ are mappings, then problem (2.1) is equivalent to finding $u \in H, x \in A u, y \in B u$, and $w \in E u$ such that $c w \in \operatorname{dom}(W)$ and

$$
0 \in a x-b y+W(c w)
$$

Variational inclusion like (2.4) have been studied in [4].

(iv) If $f=0, A=B=C=D=E=I, M(x, x)=0, N(x, x)=a x-b x$ for all $x, y \in H$, where $a, b: H \rightarrow H$ are mappings, then problem (2.1) collapses to finding $u \in H$ such that $g u-h u \in \operatorname{dom}(W)$ and

$$
0 \in a u-b u+W(g u-h u)
$$

This kind of problems have been studied in [17]. 
(v) If $f=0, C=D=I, M(x, x)=0$ for all $x \in H$, and $W=\partial \varphi$, where $\partial \varphi$ denotes the subdifferential of a proper convex lower semicontinuous function $\varphi: H \rightarrow \mathbb{R} \cup$ $\{+\infty\}$, then problem (2.1) collapses to finding $u \in H, x \in A u, y \in B u, w \in E u$ such that $g u-h w \in \operatorname{dom}(\partial \varphi)$ and

$$
\langle N(x, y), v-g u+h w\rangle \geq \varphi(g u-h w)-\varphi(v), \quad \forall v \in H
$$

which is called the generalized set-valued nonlinear quasi-variational inclusion, and studied in [14].

(vi) If $f=0, A=B=E=I, N(x, x)=g x, h x=0$ for all $x \in H$ and $W=\partial \varphi$, where $\partial \varphi$ is as above, then problem (2.1) is equivalent to finding $u \in H, x \in C u, y \in D u$ such that $g u \in \operatorname{dom}(\partial \varphi)$ and

$$
\langle g u-M(x, y), v-g u\rangle \geq \varphi(g u)-\varphi(v), \quad \forall v \in H,
$$

which is known as the multivalued mixed variational inequality, introduced and studied by M. A. Noor and K. I. Noor [12].

(vii) If $f=0, C=D=E=I, M(x, x)=h x=0$ for all $x \in H$, and $W=\partial \varphi$ where $\partial \varphi$ is as in (v), then problem (2.1) collapses to finding $u \in H, x \in A u, y \in B u$ such that $g u \in \operatorname{dom}(\partial \varphi)$ and

$$
\langle N(x, y), v-g u\rangle \geq \varphi(g u)-\varphi(v), \quad \forall v \in H,
$$

which is called the generalized multivalued mixed variational inequality, introduced and studied by M. A. Noor et al. [13].

(viii) If $f=0, C=D=E=I, M(x, x)=h x=0$ for all $x \in H, W=\partial \varphi$, where $\varphi=I_{K(u)}$, the indicator function of closed convex set $K(u)$ in $H$ defined by

$$
I_{K(u)}(x)= \begin{cases}0, & x \in K(u), \\ +\infty, & x \notin K(u),\end{cases}
$$

then problem (2.1) is equivalent to finding $u \in H, x \in A u, y \in B u$ such that $g u \in$ $K(u)$ and

$$
\langle N(x, y), v-g u\rangle \geq 0, \quad \forall v \in K(u)
$$

which is known as the generalized multivalued quasi-variational inequality, introduced and studied by M. A. Noor [9].

For appropriate and suitable choices of the mappings $g, h, A, B, C, D, E, N, M, W$, the element $f \in H$, a number of known classes of variational inequalities, quasi-variational inequalities, and quasi-variational inclusions, studied by several researchers including Aldly [1], Huang [2, 3], Jou and Yao [5], Kazmi [6], M. A. Noor [7, 8], M. A. Noor and Al-Said [11], Siddiqi and Ansari [15, 16], Uko [17], Verma [18, 19], Yao [20], and Zhang [21], can be obtained as special cases of problem (2.1). This reveals that the completely generalized multivalued nonlinear quasi-variational inclusion (2.1) is the more general and unifying one. 
Let $W: H \rightarrow 2^{H}$ be a maximal monotone mapping. Then for a given $\rho>0$, the resolvent operator associated with $W$ is defined by

$$
J_{\rho}^{W}(u)=(I+\rho W)^{-1}(u), \quad \forall u \in H .
$$

It is known that the resolvent operator $J_{\rho}^{W}$ is single-valued and nonexpansive.

Definition 2.1. A mapping $g: H \rightarrow H$ is said to be $s$-strongly monotone and $t$ Lipschitz continuous if there exist constants $s>0, t>0$ such that

$$
\langle g x-g y, x-y\rangle \geq s\|x-y\|^{2}, \quad\|g x-g y\| \leq t\|x-y\|, \quad \forall x, y \in H,
$$

respectively.

DEFINITION 2.2. A mapping $N: H \times H \rightarrow H$ is said to be $t$-Lipschitz continuous with respect to the first argument if there exists a constant $t>0$ such that

$$
\|N(x, u)-N(y, u)\| \leq t\|x-y\|, \quad \forall x, y, u \in H .
$$

In a similar way, we can define Lipschitz continuity of the mapping $N$ with respect to the second argument.

Definition 2.3. A multivalued mapping $A: H \rightarrow C B(H)$ is said to be $t$-strongly monotone with respect to the first argument of $N: H \times H \rightarrow H$, if there exists a constant $t>0$ such that

$$
\langle N(x, q)-N(y, q), u-v\rangle \geq t\|u-v\|^{2}, \quad \forall u, v, q \in H, x \in A u, y \in A v .
$$

DeFinITION 2.4. A multivalued mapping $A: H \rightarrow C B(H)$ is said to be $t$-relaxed Lipschitz with respect to the first argument of $N: H \times H \rightarrow H$, if there exists a constant $t>0$ such that

$$
\langle N(x, q)-N(y, q), u-v\rangle \leq-t\|u-v\|^{2}, \quad \forall u, v, q \in H, x \in A u, y \in A v
$$

DEFINITION 2.5. A multivalued mapping $A: H \rightarrow C B(H)$ is said to be $t$-generalized pseudocontractive with respect to the second argument of $N: H \times H \rightarrow H$, if there exists a constant $t>0$ such that

$$
\langle N(q, x)-N(q, y), u-v\rangle \leq t\|u-v\|^{2}, \quad \forall u, v, q \in H, x \in A u, y \in A v .
$$

Definition 2.6. A multivalued mapping $A: H \rightarrow C B(H)$ is said to be $t$-Lipschitz continuous, if there exists a constant $t>0$ such that

$$
H(A x, A y) \leq t\|x-y\|, \quad \forall x, y \in H
$$

3. Main results. Now we invoke the resolvent operator technique to prove that the completely generalized multivalued nonlinear quasi-variational inclusion (2.1) is equivalent to a fixed point problem. 
LEMMA 3.1. Let $\rho$ and $t$ be positive parameters. Then the following statements are equivalent:

(a) the completely generalized multivalued nonlinear quasi-variational inclusion (2.1) has a solution $u \in H, x \in A u, y \in B u, z \in C u, v \in D u, w \in E u$ with $g u-h w \in \operatorname{dom}(W)$;

(b) there exist $u \in H, x \in A u, y \in B u, z \in C u, v \in D u, w \in E u$ satisfying

$$
g u=h w+J_{\rho}^{W}(g u-h w-\rho N(x, y)+\rho M(z, v)+\rho f)
$$

(c) the multivalued mapping $G: H \rightarrow 2^{H}$ defined by

$$
\begin{aligned}
G q=\bigcup_{\substack{z \in A q, y \in B q, z \in C q, v \in D q, w \in E q}}[(1-t) q+t & (q-g q+h w \\
& +J_{\rho}^{W}(g q-h w-\rho N(x, y) \\
& +\rho M(z, v)+\rho f))], \quad \forall q \in H,
\end{aligned}
$$

has a fixed point $u \in H$.

Proof. It is evident that

$$
\begin{aligned}
f \in N & (x, y)-M(z, v)+W(g u-h w) \\
& \Longleftrightarrow g u-h w-\rho N(x, y)+\rho M(z, v)+\rho f \in(I+\rho W)(g u-h w) \\
& \Longleftrightarrow g u-h w=J_{\rho}^{W}(g u-h w-\rho N(x, y)+\rho M(z, v)+\rho f),
\end{aligned}
$$

which means that (a) and (b) are equivalent. Clearly, $u \in H$ is a fixed point of $G$ if and only if there exist $x \in A u, y \in B u, z \in C u, v \in D u$, and $w \in E u$ satisfying

$$
u=(1-t) u+t\left(u-g u+h w+J_{\rho}^{W}(g u-h w-\rho N(x, y)+\rho M(z, v)+\rho f)\right) .
$$

That is, (b) and (c) are equivalent. This completes the proof.

REMARK 3.2. Lemma 3.1 is a generalization of Lemma 3.1 in $[1,4,6,8,9,10,11,12$, 13, 14, 15, 16, 21], [2, Lemma 2.1], [3, Lemma 3.4], [5, Theorems 3.1-3.3], and [18, 19, Lemma 3.2].

Lemma 3.1 is very important from the numerical and approximation point of views. Based on Lemma 3.1 and Nadler's result, we suggest the following general and unified algorithms for the completely generalized multivalued nonlinear quasi-variational inclusion (2.1).

Algorithm 3.3. Let $g, h: H \rightarrow H, A, B, C, D, E: H \rightarrow C B(H), N, M: H \times H \rightarrow H$. For given $u_{0} \in H, x_{0} \in A u_{0}, Y_{0} \in B u_{0}, z_{0} \in C u_{0}, v_{0} \in D u_{0}$, and $w_{0} \in E u_{0}$, compute 
$\left\{u_{n}\right\}_{n \geq 0},\left\{x_{n}\right\}_{n \geq 0},\left\{y_{n}\right\}_{n \geq 0},\left\{z_{n}\right\}_{n \geq 0},\left\{v_{n}\right\}_{n \geq 0},\left\{w_{n}\right\}_{n \geq 0}$ from the iterative scheme

$$
\begin{gathered}
u_{n+1}=(1-t) u_{n}+t\left(u_{n}-g u_{n}+h w_{n}\right. \\
\left.\quad+J_{\rho}^{W}\left(g u_{n}-h w_{n}-\rho N\left(x_{n}, y_{n}\right)+\rho M\left(z_{n}, v_{n}\right)+\rho f\right)\right), \\
x_{n} \in A u_{n}, \quad\left\|x_{n}-x_{n+1}\right\| \leq\left(1+(n+1)^{-1}\right) H\left(A u_{n}, A u_{n+1}\right), \\
y_{n} \in B u_{n}, \quad\left\|y_{n}-y_{n+1}\right\| \leq\left(1+(n+1)^{-1}\right) H\left(B u_{n}, B u_{n+1}\right), \\
z_{n} \in C u_{n}, \quad\left\|z_{n}-z_{n+1}\right\| \leq\left(1+(n+1)^{-1}\right) H\left(C u_{n}, C u_{n+1}\right), \\
v_{n} \in D u_{n}, \quad\left\|v_{n}-v_{n+1}\right\| \leq\left(1+(n+1)^{-1}\right) H\left(D u_{n}, D u_{n+1}\right), \\
w_{n} \in E u_{n}, \quad\left\|w_{n}-w_{n+1}\right\| \leq\left(1+(n+1)^{-1}\right) H\left(E u_{n}, E u_{n+1}\right),
\end{gathered}
$$

for all $n \geq 0$ where $t$ and $\rho$ are positive parameters with $t \leq 1$.

Algorithm 3.4. Let $g, h: H \rightarrow H, A, B, C, D, E: H \rightarrow C B(H), N, M: H \times H \rightarrow H$. For given $u_{0} \in H, x_{0} \in A u_{0}, y_{0} \in B u_{0}, z_{0} \in C u_{0}, v_{0} \in D u_{0}$, and $w_{0} \in E u_{0}$, compute $\left\{u_{n}\right\}_{n \geq 0},\left\{x_{n}\right\}_{n \geq 0},\left\{y_{n}\right\}_{n \geq 0},\left\{z_{n}\right\}_{n \geq 0},\left\{v_{n}\right\}_{n \geq 0},\left\{w_{n}\right\}_{n \geq 0}$ from the iterative scheme

$$
g u_{n+l}=h w_{n}+J_{\rho}^{W}\left(g u_{n}-h w_{n}-\rho N\left(x_{n}, y_{n}\right)+\rho M\left(z_{n}, v_{n}\right)+\rho f\right), \quad \forall n \geq 0,
$$

where $\left\{x_{n}\right\}_{n \geq 0},\left\{y_{n}\right\}_{n \geq 0},\left\{z_{n}\right\}_{n \geq 0},\left\{v_{n}\right\}_{n \geq 0}$, and $\left\{w_{n}\right\}_{n \geq 0}$ satisfy (3.6) and $\rho$ is a positive parameter.

REMARK 3.5. Algorithms 3.3 and 3.4 include [2, Algorithm 2.1], Algorithms 3.3 and 3.4 in [3, 4, 10, 11, 14, 15, 16, 21], Algorithms 4.3 and 4.4 in [8, 9], Algorithms 4.1-4.3 in $[12,13]$, and Algorithm 3.1 in $[18,19]$ as particular cases.

Next we discuss those conditions under which the approximate solution, obtained from Algorithm 3.3 or Algorithm 3.4, converges to the exact solution of the completely generalized multivalued nonlinear quasi-variational inclusion (2.1).

THEOREM 3.6. Let $g, h: H \rightarrow H$ be a-Lipschitz continuous and b-Lipschitz continuous, respectively, and $g$ be c-strongly monotone. Let $N, M: H \times H \rightarrow H$ be $\alpha$ Lipschitz continuous and $\gamma$-Lipschitz continuous in the first arguments, respectively, and $\beta$-Lipschitz continuous and $\delta$-Lipschitz continuous in the second arguments, respectively. Suppose that $A, B, C, D, E: H \rightarrow C B(H)$ are $m$-Lipschitz continuous, $p$-Lipschitz continuous, q-Lipschitz continuous, $r$-Lipschitz continuous, and s-Lipschitz continuous, respectively, $A$ is $\xi$-strongly monotone with respect to the first argument of $N$ and $C$ is $\eta$ relaxed Lipschitz with respect to the first argument of $M$. Let $f \in H, k=2 \sqrt{1-2 c+a^{2}}+$ $2 b s, j=\beta p+\delta r, L=(\alpha m+\gamma q)^{2}-j^{2}, T=\xi+\eta-(1-k) j$, and $S=2 k-k^{2}$. If there exists a constant $\rho>0$ satisfying

$$
k+\rho j<1,
$$

and one of the following conditions:

$$
\begin{aligned}
& L>0, \quad|T|>\sqrt{S L}, \quad\left|\rho-T L^{-1}\right|<L^{-1} \sqrt{T^{2}-S L} \\
& L=0, \quad T>0, \quad \rho>(2 T)^{-1} S \\
& L<0, \quad\left|\rho-T L^{-1}\right|>(-L)^{-1} \sqrt{T^{2}-S L}
\end{aligned}
$$


then the completely generalized multivalued nonlinear quasi-variational inclusion (2.1) has a solution $u \in H, x \in A u, y \in B u, z \in C u, v \in D u, w \in E u$ with gu $-h w \in$ $\operatorname{dom}(W)$ and the sequences $\left\{u_{n}\right\}_{n \geq 0},\left\{x_{n}\right\}_{n \geq 0},\left\{y_{n}\right\}_{n \geq 0},\left\{z_{n}\right\}_{n \geq 0},\left\{v_{n}\right\}_{n \geq 0}$, and $\left\{w_{n}\right\}_{n \geq 0}$ generalized by Algorithm 3.3 converge strongly to $u, x, y, z, v$, and $w$, respectively.

Proof. Since $g$ is $a$-Lipschitz continuous and $c$-strongly monotone, it follows that

$$
\begin{aligned}
\| u_{n}- & u_{n+1}-\left(g u_{n}-g u_{n-1}\right) \|^{2} \\
& =\left\|u_{n}-u_{n-1}\right\|^{2}-2\left\langle g u_{n}-g u_{n-1}, u_{n}-u_{n-1}\right\rangle+\left\|g u_{n}-g u_{n-1}\right\|^{2} \\
& \leq\left(1-2 c+a^{2}\right)\left\|u_{n}-u_{n-1}\right\|^{2} .
\end{aligned}
$$

Note that $A$ is $m$-Lipschitz continuous and $\xi$-strongly monotone with respect to the first argument of $N, C$ is $q$-Lipschitz continuous and $\eta$-relaxed Lipschitz with respect to the first argument of $M$, and $N$ and $M$ are $\alpha$-Lipschitz continuous and $\gamma$-Lipschitz continuous with respect to the first arguments, respectively. It is easy to verify that

$$
\begin{aligned}
\| u_{n}- & u_{n-1}-\rho\left(N\left(x_{n}, y_{n}\right)-N\left(x_{n-1}, y_{n}\right)-M\left(z_{n}, v_{n}\right)+M\left(z_{n-1}, v_{n}\right)\right) \|^{2} \\
= & \left\|u_{n}-u_{n-1}\right\|^{2}-2 \rho\left\langle N\left(x_{n}, y_{n}\right)-N\left(x_{n-1}, y_{n}\right), u_{n}-u_{n-1}\right\rangle \\
& +2 \rho\left\langle M\left(z_{n}, v_{n}\right)-M\left(z_{n-1}, v_{n}\right), u_{n}-u_{n-1}\right\rangle \\
& +\rho^{2}\left\|N\left(x_{n}, y_{n}\right)-N\left(x_{n-1}, y_{n}\right)-M\left(z_{n}, v_{n}\right)+M\left(z_{n-1}, v_{n}\right)\right\|^{2} \\
\leq & {\left[1-2 \rho(\xi+\eta)+\rho^{2}(\alpha m+\gamma q)^{2}\left(1+n^{-1}\right)^{2}\right]\left\|u_{n}-u_{n-1}\right\|^{2} . }
\end{aligned}
$$

Using (3.5), (3.6), (3.10), and (3.11), the nonexpansivity of $J_{\rho}^{W}$, the Lipschitz continuity of $B, D, E$, and the Lipschitz continuity of $N$ and $M$ with respect to the second arguments, we know that

$$
\begin{aligned}
\left\|u_{n+1}-u_{n}\right\| \leq & (1-t)\left\|u_{n}-u_{n-1}\right\|+t\left\|u_{n}-u_{n-1}-\left(g u_{n}-g u_{n-1}\right)\right\|+t\left\|h w_{n}-h w_{n-1}\right\| \\
& +t \| J_{\rho}^{W}\left(g u_{n}-h w_{n}-\rho N\left(x_{n}, y_{n}\right)+\rho M\left(z_{n}, v_{n}\right)+\rho f\right) \\
& \quad-J_{\rho}^{W}\left(g u_{n-1}-h w_{n-1}-\rho N\left(x_{n-1}, y_{n-1}\right)+\rho M\left(z_{n-1}, v_{n-1}\right)+\rho f\right) \| \\
\leq & (1-t)\left\|u_{n}-u_{n-1}\right\|+2 t\left\|u_{n}-u_{n-1}-\left(g u_{n}-g u_{n-1}\right)\right\|+2 t\left\|h w_{n}-h w_{n-1}\right\| \\
& +t\left\|u_{n}-u_{n-1}-\rho\left(N\left(x_{n}, y_{n}\right)-N\left(x_{n-1}, y_{n}\right)-M\left(z_{n}, v_{n}\right)+M\left(z_{n-1}, v_{n}\right)\right)\right\| \\
& +t \rho\left\|N\left(x_{n-1}, y_{n}\right)-N\left(x_{n-1}, y_{n-1}\right)\right\|+t \rho\left\|M\left(z_{n-1}, v_{n}\right)-M\left(z_{n-1}, v_{n-1}\right)\right\| \\
\leq & \left(1-\left(1-\theta_{n}\right) t\right)\left\|u_{n}-u_{n-1}\right\|,
\end{aligned}
$$

where

$$
\begin{aligned}
\theta_{n}= & 2 \sqrt{1-2 c+a^{2}}+2 b s\left(1+n^{-1}\right) \\
& +\sqrt{1-2 \rho(\xi+\eta)+\rho^{2}(\alpha m+\gamma q)^{2}\left(1+n^{-1}\right)^{2}} \\
& +\rho(\beta p+\delta r)\left(1+n^{-1}\right) \\
& \rightarrow \theta=k+\sqrt{1-2 \rho(\xi+\eta)+\rho^{2}(\alpha m+\gamma q)^{2}}+\rho j,
\end{aligned}
$$


as $n \rightarrow \infty$. Equation (3.8) ensures that

$$
\begin{aligned}
\theta<1 & \Leftrightarrow \sqrt{1-2 \rho(\xi+\eta)+\rho^{2}(\alpha m+\gamma q)^{2}}<1-k-\rho j \\
& \Longleftrightarrow L \rho^{2}-2 \rho T<-S .
\end{aligned}
$$

It follows from (3.14) and one of (3.9) that $\theta<1$. Let $P=2^{-1}(1+\theta)$. From (3.13) we conclude that there exists a positive integer $N_{0}$ such that $\theta_{n}<P<1$ for all $n \geq N_{0}$. Thus (3.12) ensures that

$$
\left\|u_{n+1}-u_{n}\right\| \leq(1-(1-P) t)\left\|u_{n}-u_{n-1}\right\|, \quad \forall n \geq N_{0} .
$$

Since $t \in(0,1]$, (3.15) yields that $\left\{u_{n}\right\}_{n \geq 0}$ is a Cauchy sequence in $H$. In view of (3.6) and the Lipschitz continuity of $A, B, C, D$, and $E$, we obtain that $\left\{x_{n}\right\}_{n \geq 0},\left\{y_{n}\right\}_{n \geq 0}$, $\left\{z_{n}\right\}_{n \geq 0},\left\{v_{n}\right\}_{n \geq 0},\left\{w_{n}\right\}_{n \geq 0}$ are Cauchy sequences in $H$. Let $u_{n} \rightarrow u \in H, x_{n} \rightarrow x \in H$, $y_{n} \rightarrow y \in H, z_{n} \rightarrow z \in H, v_{n} \rightarrow v \in H$, and $w_{n} \rightarrow w \in H$ as $n \rightarrow \infty$. Observe that

$$
\begin{aligned}
d(x, A u) & =\inf \{\|x-l\|: l \in A u\} \\
& \leq\left\|x-x_{n}\right\|+H\left(A u_{n}, A u\right) \longrightarrow 0 \text { as } n \longrightarrow \infty,
\end{aligned}
$$

which implies that $x \in A u$. Similarly, we can prove that $y \in B u, z \in C u, v \in D u$, and $w \in E u$. It follows from (3.5) that

$$
u=(1-t) u+t\left(u-g u+h w+J_{\rho}^{W}(g u-h w-\rho N(x, y)+\rho M(z, v)+\rho f)\right) .
$$

By virtue of Lemma 3.1, we see that the completely generalized multivalued nonlinear quasi-variational inclusion (2.1) has a solution $u \in H, x \in A u, y \in B u, z \in C u, v \in$ $D u$, and $w \in E u$. This completes the proof.

Theorem 3.7. Let $g, h, N, M, A, B, C, D, E, k, S$ be as in Theorem 3.6. Suppose that $B$ is $\zeta$-generalized pseudocontractive with respect to the second argument of $N, j=\sqrt{1+2 \zeta+\beta^{2} p^{2}}+\sqrt{1-2 \eta+\gamma^{2} q^{2}}+\delta r, L=\alpha^{2} m^{2}-j^{2}$, and $T=\xi-(1-k) j$. If there exists a constant $\rho>0$ satisfying (3.8) and one of (3.9), then the completely generalized multivalued nonlinear quasi-variational inclusion (2.1) has a solution $u \in H$, $x \in A u, y \in B u, z \in C u, v \in D u, w \in E u$ with $g u-h w \in \operatorname{dom}(W)$ and the sequences $\left\{u_{n}\right\}_{n \geq 0},\left\{x_{n}\right\}_{n \geq 0},\left\{y_{n}\right\}_{n \geq 0},\left\{z_{n}\right\}_{n \geq 0},\left\{v_{n}\right\}_{n \geq 0}$, and $\left\{w_{n}\right\}_{n \geq 0}$ generalized by Algorithm 3.3 converge strongly to $u, x, y, z, v$, and $w$, respectively.

Proof. Notice that $B$ is $p$-Lipschitz continuous and $\zeta$-generalized pseudocontractive with respect to the second argument of $N$, and $N$ is $\beta$-Lipschitz continuous in the second argument. It follows that

$$
\begin{aligned}
\| u_{n}- & u_{n-1}+N\left(x_{n-1}, y_{n}\right)-N\left(x_{n-1}, y_{n-1}\right) \|^{2} \\
= & \left\|u_{n}-u_{n-1}\right\|^{2}+2\left\langle N\left(x_{n-1}, y_{n}\right)-N\left(x_{n-1}, y_{n-1}\right), u_{n}-u_{n-1}\right\rangle \\
& +\left\|N\left(x_{n-1}, y_{n}\right)-N\left(x_{n-1}, y_{n-1}\right)\right\|^{2} \\
\leq & \left(1+2 \zeta+\beta^{2} p^{2}\left(1+n^{-1}\right)^{2}\right)\left\|u_{n}-u_{n-1}\right\|^{2} .
\end{aligned}
$$


Similarly, we have

$$
\begin{gathered}
\left\|u_{n}-u_{n-1}-\rho\left(N\left(x_{n}, y_{n}\right)-N\left(x_{n-1}, y_{n}\right)\right)\right\| \\
\leq \sqrt{1-2 \rho \xi+\rho^{2} \alpha^{2} m^{2}\left(1+n^{-1}\right)^{2}}\left\|u_{n}-u_{n-1}\right\|, \\
\left\|u_{n}-u_{n-1}+M\left(z_{n}, v_{n}\right)-M\left(z_{n-1}, v_{n}\right)\right\| \\
\leq \sqrt{1-2 \eta+\gamma^{2} q^{2}\left(1+n^{-1}\right)^{2}}\left\|u_{n}-u_{n-1}\right\| .
\end{gathered}
$$

From (3.5), (3.6), (3.10), (3.18), and (3.19), we get that

$$
\begin{aligned}
\left\|u_{n+1}-u_{n}\right\| \leq & (1-t)\left\|u_{n}-u_{n-1}\right\|+t\left\|u_{n}-u_{n-1}-\left(g u_{n}-g u_{n-1}\right)\right\|+t\left\|h w_{n}-h w_{n-1}\right\| \\
& +t \| J_{\rho}^{W}\left(g u_{n}-h w_{n}-\rho N\left(x_{n}, y_{n}\right)+\rho M\left(z_{n}, v_{n}\right)+\rho f\right) \\
& \quad-J_{\rho}^{W}\left(g u_{n-1}-h w_{n-1}-\rho N\left(x_{n-1}, y_{n-1}\right)+\rho M\left(z_{n-1}, v_{n-1}\right)+\rho f\right) \| \\
\leq & (1-t)\left\|u_{n}-u_{n-1}\right\|+2 t\left\|u_{n}-u_{n-1}-\left(g u_{n}-g u_{n-1}\right)\right\| \\
& +2 t\left\|h w_{n}-h w_{n-1}\right\|+t\left\|u_{n}-u_{n-1}-\rho\left(N\left(x_{n}, y_{n}\right)-N\left(x_{n-1}, y_{n}\right)\right)\right\| \\
& +t \rho\left\|u_{n}-u_{n-1}+N\left(x_{n-1}, y_{n}\right)-N\left(x_{n-1}, y_{n-1}\right)\right\| \\
& +t \rho\left\|u_{n}-u_{n-1}+M\left(z_{n}, v_{n}\right)-M\left(z_{n-1}, v_{n}\right)\right\| \\
& +t \rho\left\|M\left(z_{n-1}, v_{n}\right)-M\left(z_{n-1}, v_{n-1}\right)\right\| \\
\leq & \left(1-\left(1-\theta_{n}\right) t\right)\left\|u_{n}-u_{n-1}\right\|,
\end{aligned}
$$

where

$$
\begin{aligned}
\theta_{n}= & 2 \sqrt{1-2 c+a^{2}}+2 b s\left(1+n^{-1}\right)+\sqrt{1-2 \rho \xi+\rho^{2} \alpha^{2} m^{2}\left(1+n^{-1}\right)^{2}} \\
& +\rho\left(\sqrt{1+2 \zeta+\beta^{2} p^{2}\left(1+n^{-1}\right)^{2}}+\sqrt{1-2 \eta+\gamma^{2} q^{2}\left(1+n^{-1}\right)^{2}}+\delta r\left(1+n^{-1}\right)\right) \\
& \rightarrow \theta=k+\sqrt{1-2 \rho \xi+\rho^{2} \alpha^{2} m^{2}}+\rho j,
\end{aligned}
$$

as $n \rightarrow \infty$. By a similar argument used in the proof of Theorem 3.6, the result follows. This completes the proof.

REMARK 3.8. Theorems 3.6 and 3.7 extend Theorem 3.1 in [2, 15, 16, 21], Theorems 4.1 and 4.2 in $[3,4,5,14]$, and Theorem 4.1 in $[12,13]$ in the following ways:

(i) the set-valued nonlinear generalized variational inclusion in [2], the completely generalized strongly nonlinear implicit quasi-variational inequality and the generalized strongly nonlinear implicit quasi-variational inequality in [3], the variational inclusions in [4], the generalized multivalued variational inequality in [5], the multivalued mixed variational inequality in [12], the generalized multivalued mixed variational inequality in [13], the generalized set-valued strongly nonlinear quasi-variational inclusion and the generalized set-valued nonlinear quasi-variational inclusion in [14], the strongly nonlinear variational inequality in [15], the general strongly nonlinear variational inequality in [16], and the general set-valued strongly nonlinear quasivariational inequality in [21] involving strongly monotone mappings are replaced by 
the more general completely generalized multivalued nonlinear quasi-variational inclusion involving strongly monotone mappings, relaxed Lipschitz mappings, and generalized pseudocontractive mappings.

(ii) [2, Algorithm 2.1], Algorithms 3.1 and 3.2 in [3, 4, 14, 15, 16, 21], Algorithms 4.1-4.3 in [12, 13] are replaced by the more general Algorithm 3.3.

(iii) Conditions (3.9) are weaker than the conditions used in [2, 3, 4, 5, 12, 13, 14, $15,16,21]$.

TheOrem 3.9. Let $g, h, N, M, A, B, C, D, E, f, j, L$ be as in Theorem 3.6. Let $c \leq 1$, $k=\sqrt{1-2 c+a^{2}}+2 b s, T=\xi+\eta-(c-k) j$, and $S=1-(c-k)^{2}$. If there exists a constant $\rho>0$ satisfying

$$
k+\rho j<c,
$$

and one of (3.9), then the completely generalized multivalued nonlinear quasi-variational inclusion (2.1) has a solution $u \in H, x \in A u, y \in B u, z \in C u, v \in D u, w \in$ Eu with $g u-h w \in \operatorname{dom}(W)$ and the sequences $\left\{u_{n}\right\}_{n \geq 0},\left\{x_{n}\right\}_{n \geq 0},\left\{y_{n}\right\}_{n \geq 0},\left\{z_{n}\right\}_{n \geq 0}$, $\left\{v_{n}\right\}_{n \geq 0}$, and $\left\{w_{n}\right\}_{n \geq 0}$ generalized by Algorithm 3.4 converge strongly to $u, x, y, z$, $v$, and $w$, respectively.

Proof. Using the strong monotonicity of $g$, (3.7), (3.10), and (3.11), we infer that

$$
\begin{aligned}
\| u_{n+1} & -u_{n} \| \\
\leq & c^{-1}\left\|g u_{n+1}-g u_{n}\right\| \leq c^{-1}\left\|h w_{n}-h w_{n-1}\right\| \\
& +c^{-1} \| J_{\rho}^{W}\left(g u_{n}-h w_{n}-\rho N\left(x_{n}, y_{n}\right)+\rho M\left(z_{n}, v_{n}\right)+\rho f\right) \\
& \quad-J_{\rho}^{W}\left(g u_{n-1}-h w_{n-1}-\rho N\left(x_{n-1}, y_{n-1}\right)+\rho M\left(z_{n-1}, v_{n-1}\right)+\rho f\right) \| \\
\leq & 2 c^{-1}\left\|h w_{n}-h w_{n-1}\right\|+c^{-1}\left\|u_{n}-u_{n-1}-\left(g u_{n}-g u_{n-1}\right)\right\| \\
& +c^{-1}\left\|u_{n}-u_{n-1}-\rho\left(N\left(x_{n}, y_{n}\right)-N\left(x_{n-1}, y_{n}\right)-M\left(z_{n}, v_{n}\right)+M\left(z_{n-1}, v_{n}\right)\right)\right\| \\
& +c^{-1} \rho\left(\left\|N\left(x_{n-1}, y_{n}\right)-N\left(x_{n-1}, y_{n-1}\right)\right\|+\left\|M\left(z_{n-1}, v_{n}\right)-M\left(z_{n-1}, v_{n-1}\right)\right\|\right) \\
\leq & \theta_{n}\left\|u_{n}-u_{n-1}\right\|,
\end{aligned}
$$

where

$$
\begin{aligned}
\theta_{n}=c^{-1}[ & 2 b s\left(1+n^{-1}\right)+\sqrt{1-2 c+a^{2}} \\
& \left.+\sqrt{1-2 \rho(\xi+\eta)+\rho^{2}(\alpha m+\gamma q)^{2}\left(1+n^{-1}\right)^{2}}+\rho(\beta p+\delta r)\left(1+n^{-1}\right)\right] \\
\longrightarrow \theta= & c^{-1}\left(k+\sqrt{1-2 \rho(\xi+\eta)+\rho^{2}(\alpha m+\gamma q)^{2}}+\rho j\right),
\end{aligned}
$$

as $n \rightarrow \infty$. The rest of the argument is the same as in the proof of Theorem 3.6 and is therefore omitted. This completes the proof.

TheOrem 3.10. Let $g, h, M, A, B, C, D, E, f, c, k, S$ be as in Theorem $3.9, B, L, j$ be as in Theorem 3.7. Let $T=\xi-(c-k) j$. If there exists a constant $\rho>0$ satisfying (3.22) and 
one of (3.9), then the completely generalized multivalued nonlinear quasi-variational inclusion (2.1) has a solution $u \in H, x \in A u, y \in B u, z \in C u, v \in D u, w \in E u$ with $g u-h w \in \operatorname{dom}(W)$ and the sequences $\left\{u_{n}\right\}_{n \geq 0},\left\{x_{n}\right\}_{n \geq 0},\left\{y_{n}\right\}_{n \geq 0},\left\{z_{n}\right\}_{n \geq 0},\left\{v_{n}\right\}_{n \geq 0}$, and $\left\{w_{n}\right\}_{n \geq 0}$ generalized by Algorithm 3.4 converge strongly to $u, x, y, z, v$, and $w$, respectively.

Proof. As in the proofs of Theorems 3.7 and 3.9, we know that

$$
\begin{aligned}
\| u_{n+1} & -u_{n} \| \\
\leq & c^{-1}\left\|h w_{n}-h w_{n-1}\right\| \\
& +c^{-1} \| J_{\rho}^{W}\left(g u_{n}-h w_{n}-\rho N\left(x_{n}, y_{n}\right)+\rho M\left(z_{n}, v_{n}\right)+\rho f\right) \\
& \quad-J_{\rho}^{W}\left(g u_{n-1}-h w_{n-1}-\rho N\left(x_{n-1}, y_{n-1}\right)+\rho M\left(z_{n-1}, v_{n-1}\right)+\rho f\right) \| \\
\leq & 2 c^{-1}\left\|h w_{n}-h w_{n-1}\right\|+c^{-1}\left\|u_{n}-u_{n-1}-\left(g u_{n}-g u_{n-1}\right)\right\| \\
& +c^{-1}\left\|u_{n}-u_{n-1}-\rho\left(N\left(x_{n}, y_{n}\right)-N\left(x_{n-1}, y_{n}\right)\right)\right\| \\
& +c^{-1} \rho\left\|u_{n}-u_{n-1}+N\left(x_{n-1}, y_{n}\right)-N\left(x_{n-1}, y_{n-1}\right)\right\| \\
& +c^{-1} \rho\left\|u_{n}-u_{n-1}+M\left(z_{n}, v_{n}\right)-M\left(z_{n-1}, v_{n}\right)\right\| \\
& +c^{-1} \rho\left\|M\left(z_{n-1}, v_{n}\right)-M\left(z_{n-1}, v_{n-1}\right)\right\| \\
\leq & \theta_{n}\left\|u_{n}-u_{n-1}\right\|,
\end{aligned}
$$

where

$$
\begin{aligned}
\theta_{n}=c^{-1}[ & 2 b s\left(1+n^{-1}\right)+\sqrt{1-2 c+a^{2}}+\sqrt{1-2 \rho \xi+\rho^{2} \alpha^{2} m^{2}\left(1+n^{-1}\right)^{2}} \\
& \left.+\rho\left(\sqrt{1+2 \zeta+\beta^{2} p^{2}\left(1+n^{-1}\right)^{2}}+\sqrt{1-2 \eta+\gamma^{2} q^{2}\left(1+n^{-1}\right)^{2}}+\delta r\left(1+n^{-1}\right)\right)\right] \\
\longrightarrow \theta= & c^{-1}\left(k+\sqrt{1-2 \rho \xi+\rho^{2} \alpha^{2} m^{2}}+\rho j\right),
\end{aligned}
$$

as $n \rightarrow \infty$. The rest of the proof follows precisely as in the proof of Theorem 3.6. This completes the proof.

REMARK 3.11. Theorems 3.9 and 3.10 extend, improve, and unify Theorem 3.1 in $[18,19]$ and [20, Theorem 3.6].

ACKNOWLEDGMENT. This work was supported by Korea Research Foundation Grant (KRF-2000-DP0013).

\section{REFERENCES}

[1] S. Adly, Perturbed algorithms and sensitivity analysis for a general class of variational inclusions, J. Math. Anal. Appl. 201 (1996), no. 2, 609-630.

[2] N. J. Huang, Generalized nonlinear variational inclusions with noncompact valued mappings, Appl. Math. Lett. 9 (1996), no. 3, 25-29.

[3] _ On the generalized implicit quasivariational inequalities, J. Math. Anal. Appl. 216 (1997), no. 1, 197-210. 
[4] _ A new completely general class of variational inclusions with noncompact valued mappings, Comput. Math. Appl. 35 (1998), no. 10, 9-14.

[5] C. R. Jou and J. C. Yao, Algorithm for generalized multivalued variational inequalities in Hilbert spaces, Comput. Math. Appl. 25 (1993), no. 9, 7-16.

[6] K. R. Kazmi, Mann and Ishikawa type perturbed iterative algorithms for generalized quasivariational inclusions, J. Math. Anal. Appl. 209 (1997), no. 2, 572-584.

[7] M. A. Noor, Generalized multivalued variational inequalities. II, Comput. Math. Appl. 30 (1995), no. 11, 83-89.

[8] _ Generalized multivalued quasi-variational inequalities, Comput. Math. Appl. 31 (1996), no. 12, 1-13.

[9]___ Generalized multivalued quasi-variational inequalities. II, Comput. Math. Appl. 35 (1998), no. 5, 63-78.

[10] _ Generalized set-valued variational inclusions and resolvent equations, J. Math. Anal. Appl. 228 (1998), no. 1, 206-220.

[11] M. A. Noor and E. A. Al-Said, Iterative methods for generalized nonlinear variational inequalities, Comput. Math. Appl. 33 (1997), no. 8, 1-11.

[12] M. A. Noor and K. I. Noor, Multivalued variational inequalities and resolvent equations, Math. Comput. Modelling 26 (1997), no. 7, 109-121.

[13] M. A. Noor, K. I. Noor, and T. M. Rassias, Set-valued resolvent equations and mixed variational inequalities, J. Math. Anal. Appl. 220 (1998), no. 2, 741-759.

[14] S. H. Shim, S. M. Kang, N. J. Hung, and Y. J. Cho, Generalized set-valued strongly nonlinear quasivariational inclusions, Indian J. Pure Appl. Math. 31 (2000), no. 9, 1113-1122.

[15] A. H. Siddiqi and Q. H. Ansari, Strongly nonlinear quasivariational inequalities, J. Math. Anal. Appl. 149 (1990), no. 2, 444-450.

[16]__ General strongly nonlinear variational inequalities, J. Math. Anal. Appl. 166 (1992), no. 2, 386-392.

[17] L. U. Uko, Strongly nonlinear generalized equations, J. Math. Anal. Appl. 220 (1998), no. 1, 65-76.

[18] R. U. Verma, Iterative algorithms for variational inequalities and associated nonlinear equations involving relaxed Lipschitz operators, Appl. Math. Lett. 9 (1996), no. 4, 61-63.

[19] —_, Generalized variational inequalities and associated nonlinear equations, Czechoslovak Math. J. 48(123) (1998), no. 3, 413-418.

[20] J. C. Yao, Applications of variational inequalities to nonlinear analysis, Appl. Math. Lett. 4 (1991), no. 4, 89-92.

[21] J. H. Zhang, General strongly nonlinear variational inequalities for multifunctions, Appl. Math. Lett. 8 (1995), no. 3, 75-80.

Zeqing Liu: Department of MATHematics, LiaOning Normal University, Dalian, LIAONING, 116029, CHINA

E-mail address: zeqingliu@sina.com.cn

LOKENATH Debnath: DePARTMENT OF MATHEMATICS, UNIVERSiTy OF TEXAS, PAN AMERICAN, EDINBURG, TEXAS 78539, USA

E-mail address: debnath1@panam.edu

Shin Min Kang: Department of Mathematics, Gyeongsang National University, CHINJU 660-701, KOREA

E-mail address: smkang@nongae.gsnu.ac.kr

Jeong Sheok Ume: Department of ApPlied Mathematics, Changwon NATIONAL UniverSITY, CHANGWON 641-773, KOREA

E-mail address: jsume@sarim.changwon.ac.kr 


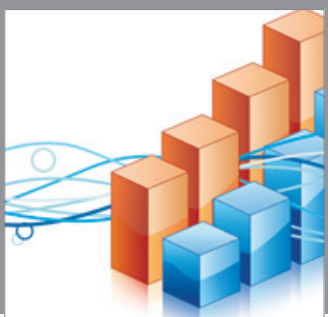

Advances in

Operations Research

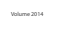

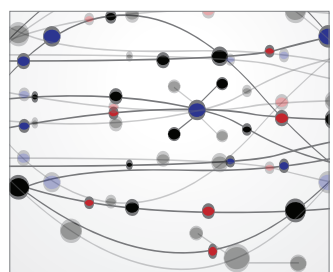

\section{The Scientific} World Journal
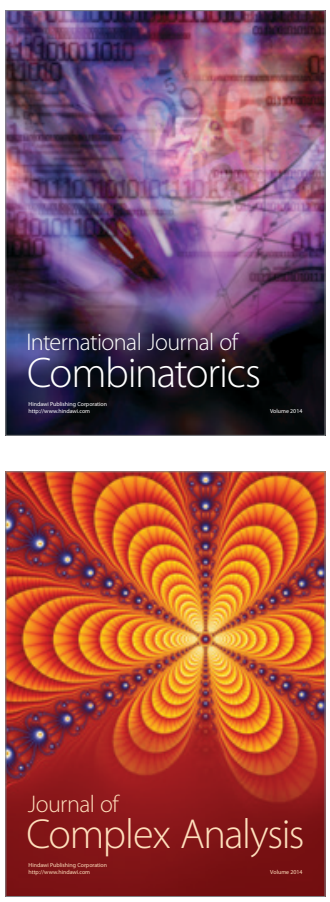

International Journal of

Mathematics and

Mathematical

Sciences
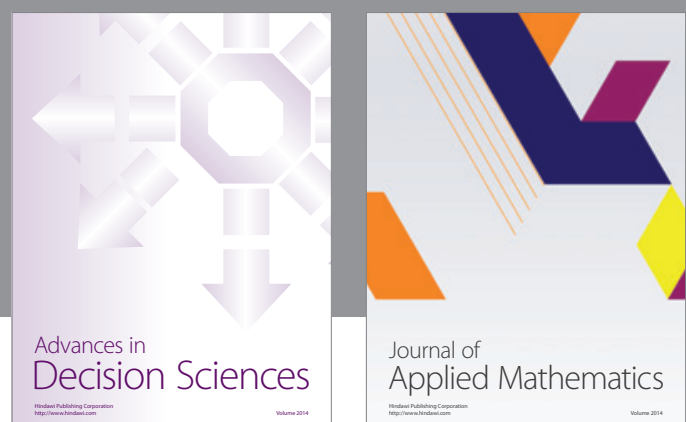

Journal of

Applied Mathematics
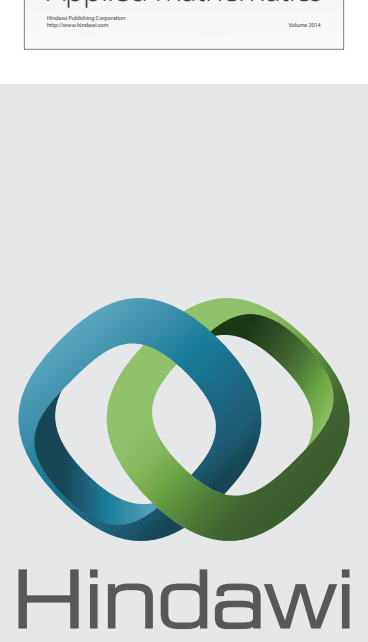

Submit your manuscripts at http://www.hindawi.com
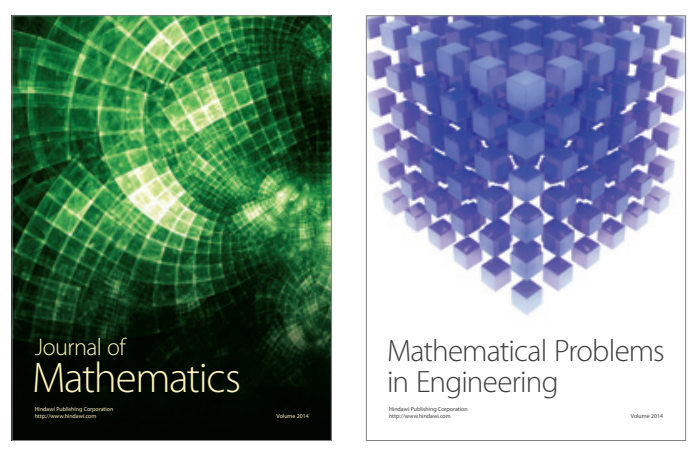

Mathematical Problems in Engineering
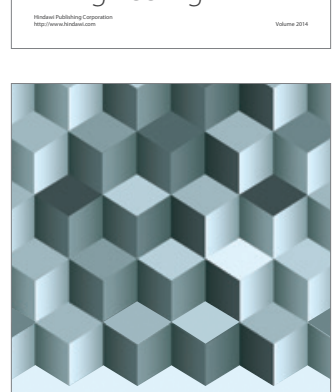

Journal of

Function Spaces
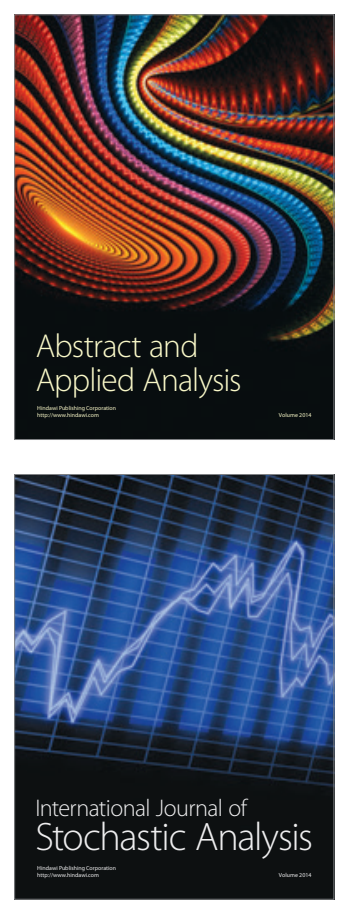

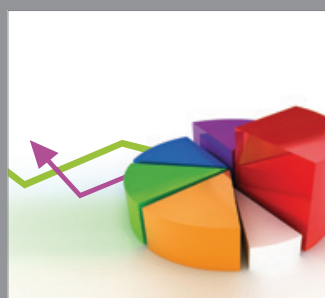

ournal of

Probability and Statistics

Promensencen
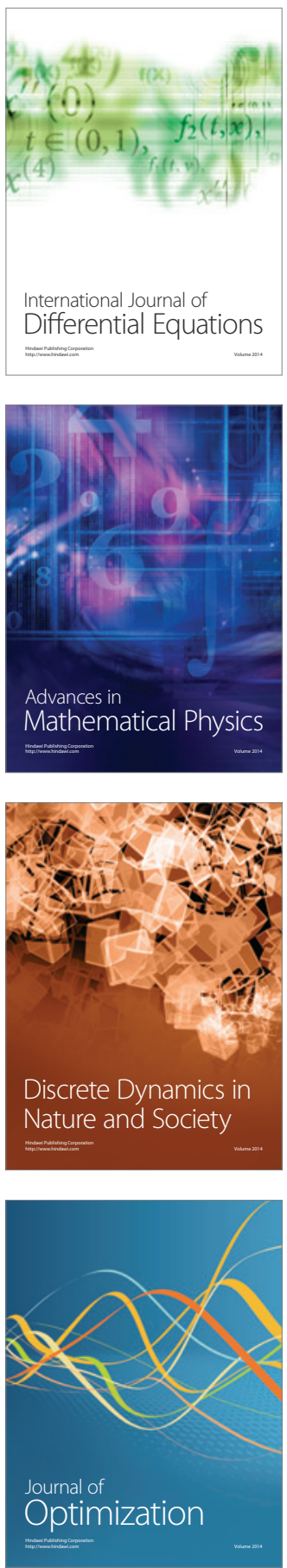